\title{
Study and monitoring of the stability of a rock mass at the Dar-El-Oued tunnel, wilaya of Jijel - Algeria
}

\section{Étude et suivi de la stabilité d'un massif rocheux au niveau du tunnel de Dar-El-Oued, wilaya de Jijel - Algérie}

\author{
Chahra YELLAS ${ }^{1}$, Riad BENZAID ${ }^{2}$, Mustapha TEKKOUK ${ }^{3}$ \\ 1,2,3 Laboratoire de Génie Géologique, Université Mohammed Seddik Benyahia, Jijel, Algérie.
}

\begin{abstract}
The construction of a civil engineering work requires many preliminary studies as well as various calculation methods to give an assessment or a forecast of the state of the rock in place and the behavior of the structure in its real environment and under various conditions (construction phase, service and operating phase). The present work concerns the access zone of the Dar-El-Oued tunnel, located on the national road $\mathrm{n}^{\circ} 43$ about $40 \mathrm{~km}$ West of the city of Jijel (northeastern Algeria). The geotechnical study of this tunnel was carried out following the classification methods relating to underground structures. Through the results of this study we have determined the influence of land pressures on the mechanical behavior of the ground, which allowed us to measure the state of stress in the rock and to make a comparison between the results of the measurements of convergence and that of numerical modeling. On the other hand, the influence of the velocity of vibration induced by explosive shots has been discussed in order to check the stability of the face as the digging progresses. From the results obtained, it can be said that the surface displacements of the soil are relatively small compared to those determined by the numerical model at the level of the vault and the slab. Note the good agreement between the results provided by our numerical model and those obtained in-situ.
\end{abstract}

\begin{abstract}
Résumé. La construction d'un ouvrage de génie civil nécessite de nombreuses études préliminaires ainsi que diverses méthodes de calcul pour donner une évaluation ou une prévision de l'état de la roche en place et du fonctionnement de l'ouvrage dans son environnement réel et sous diverses sollicitations (phase de construction, phase de service et d'exploitation). Le présent travail concerne le front de taille du tunnel de Dar-El-Oued, situé sur la route nationale $\mathrm{n}^{\circ} 43$ à environ $40 \mathrm{~km}$ à l'ouest de la ville de Jijel (Nord-Est Algérien). L'étude géotechnique de ce tunnel a été réalisée en suivant les méthodes de classifications relatives aux ouvrages souterrains. A travers les résultats de cette étude nous avons déterminé l'influence des pressions des terres sur le comportement mécanique du terrain, ce qui nous a permis de mesurer l'état de contrainte dans la roche et de faire une comparaison entre les résultats des mesures de convergence et celle de la modélisation numérique. D'autre part, l'influence de la vitesse de vibration induite par les tirs d'explosifs a été discutée dans le but de vérifier la stabilité du front de taille au fur et à mesure de l'avancement des travaux de creusement. A partir des résultats obtenus, on peut dire que les déplacements en surface du sol sont relativement faibles par rapport à ceux déterminés par le modèle numérique au niveau de la voûte et du radier. Notons ainsi la bonne concordance entre les résultats prévus par notre modèle numérique et ceux des mesures obtenues in-situ.
\end{abstract}

\section{Introduction}

Le projet du tunnel de Dar El-Oued s'inscrit dans les travaux d'adaptation fonctionnelle de la route côtière qui relie la ville de Jijel à la ville de Bejaia (Nord-Est Algérien). Il est né de l'exigence d'éviter le tronçon de l'actuelle route côtière dont la section est sensiblement réduite à proximité des fameuses grottes merveilleuses (Figure 1).

L'ajustement de la Grotte Merveilleuse, ainsi que tout le versant à pic sur la mer, à lui seul de grande valeur 
touristique. D'où la décision de créer un nouveau tunnel, en amont du tracé actuel, qui perce le Djebel Hamra du côté mer, en évitant ainsi les Grottes Merveilleuses et le tronçon de côte attenant.

Les travaux de creusement du tunnel ont provoqué une modification de l'état de contraintes dans la roche autour de la galerie. Ce changement d'état de contraintes est à l'origine des déplacements, qui affectent le terrain avoisinant et les structures préexistantes, particulièrement sensible, sachant que ce passage d'un état d'équilibre à un autre n'est pas instantané.

Les travaux de creusement ont provoqué une succession d'instabilités intermédiaires, qui influencent l'état d'équilibre final. Quelle que soit la technique de construction employée, il se produit toujours des déplacements autour de la galerie, qui peuvent se propager dans le massif et atteindre la surface du sol. Ces déplacements dépendent des caractéristiques du sol dans chaque section, des conditions géométriques et de la technique de creusement. C'est pourquoi il est important de localiser les déformations et les déplacements associés au creusement.

Dans le souci d'assurer une sécurité optimale pour le projet de construction du tunnel de Dar El-Oued, les pressions sur le soutènement ont étaient calculées par plusieurs méthodes théoriques, en plus d'une analyse basée sur une modélisation numérique par éléments finis en utilisant le logiciel SAP 2000.

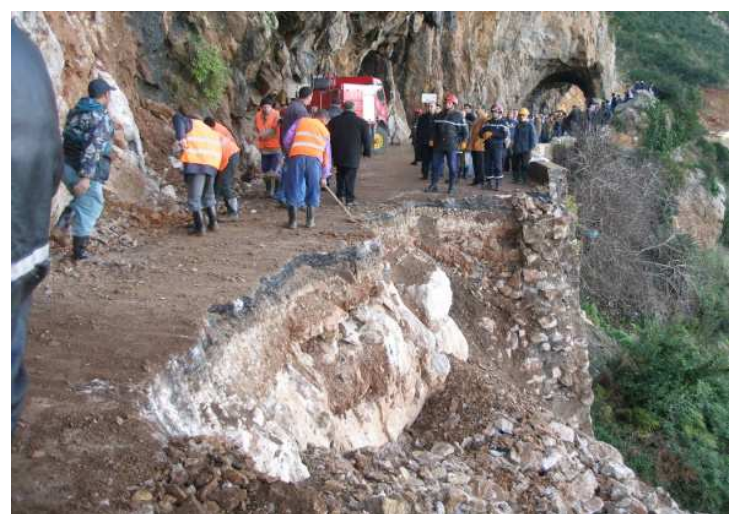

Fig. 1. L'ancien tracé de la route à proximité du site des Grottes Merveilleuses

\section{Description du tunnel de Dar El-Oued}

Cet ouvrage se situe sur la route nationale $n^{\circ} 43$ (RN43) à environ $40 \mathrm{~km}$ à l'Ouest de la ville de Jijel (Nord-Est Algérien) au niveau du massif carbonaté de Dar-El-Oued, bordé au Nord par la mer Méditerranée et à l'Est par l'oued de Dar-El-Oued (Figure 2). Le tracé du tunnel se prolonge sur une longueur de $620 \mathrm{~m}$ creusé dans les calcaires dolomitiques d'âge Jurassique. Il est constitué de deux voies de 3,50 m de large chacune et des accotements de 1,40 m de large, avec un gabarit en hauteur de $5 \mathrm{~m}$ environ. Un système de drainage rigoureux été réalisé sur toute la longueur de la galerie pour assurer une bonne étanchéité du tunnel. A l'entrée Est, un faux tunnel sur $5 \mathrm{ml}$ de long été réalisé pour faciliter le début des travaux. Par ailleurs l'embouchure
Ouest est protégée par une berlinoise en micro pieux renforcée avec des tirants d'ancrages.
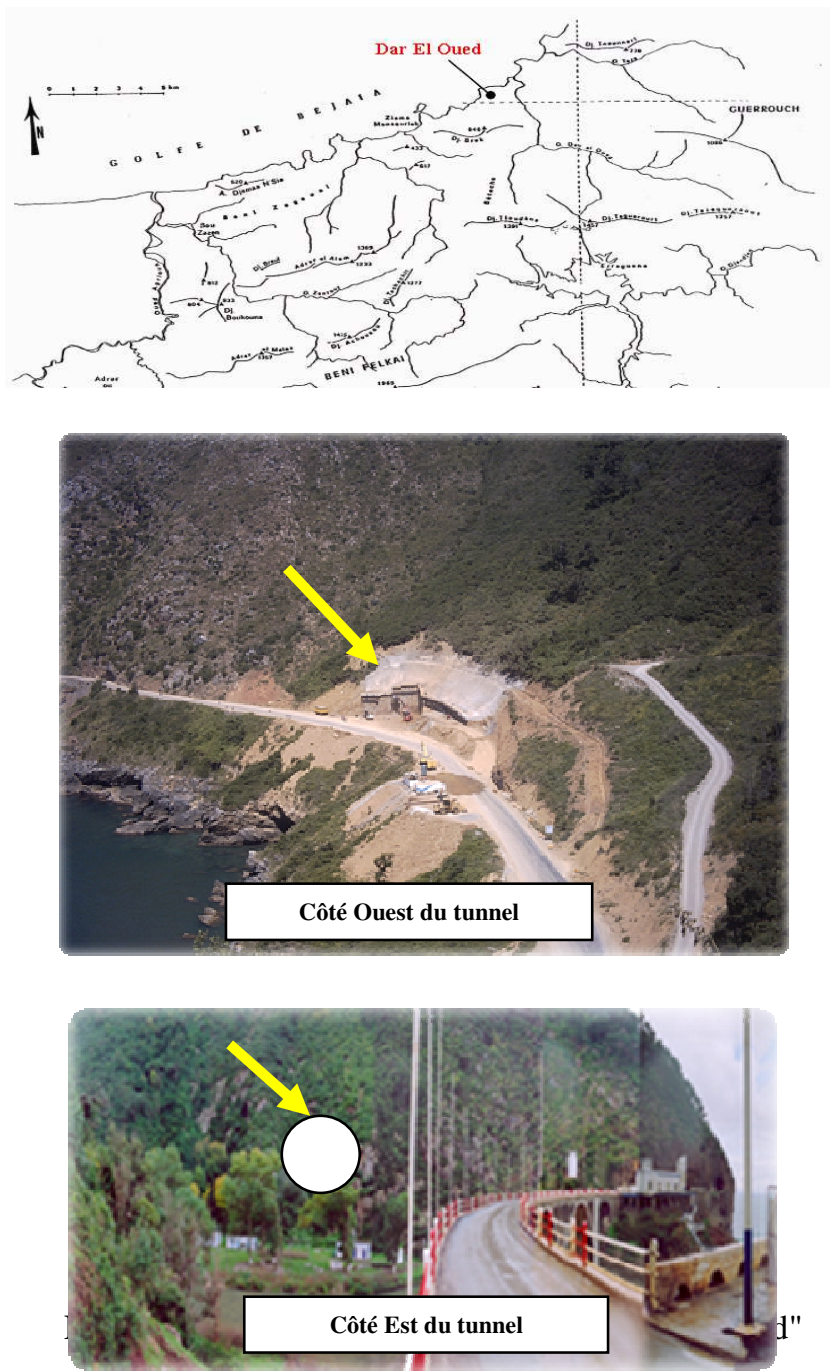

\section{Influence de la vitesse de vibration sur la stabilité du front de taille}

Pour prédire les effets d'un tir en un point donné de la section du tunnel, il convient de déterminer la relation entre la quantité d'explosif utilisée et la vitesse de vibration résultante au point considéré, ce qui permet d'adapter le plan de tir à l'environnement. La seule façon appropriée de définir cette relation et de prévoir sur le site des tirs expérimentaux où l'on fait croître la charge toute en mesurant la vitesse de vibration correspondante $[1,2]$. En avant-projet et à défaut de tirs expérimentaux, il est toujours possible d'utiliser des relations empiriques. Les formules publiées sont diverses et donnent des résultats souvent très différents. En effet, les relations établies ne font pas apparaître les facteurs pourtant importants que sont la nature du rocher et la géométrie du plan de tir. On peut néanmoins retenir en toute première approximation la formule proposée par Chapot (1981) [3] pour les tirs en tunnels :

$$
\mathrm{V}=\mathrm{K} \cdot \mathrm{Q}^{0,9} / \mathrm{D}^{1,8}
$$


Avec, V vitesse particulaire en $(\mathrm{cm} / \mathrm{s})$; D distance entre le point de mesure et le point de tir en (m) et Q la quantité d'explosif en ( $\mathrm{gr})$.

Le paramètre $\mathrm{K}$ varie suivant le site et la nature de la roche. Pour les tirs en tunnel, on peut retenir à titre indicatif les valeurs suivantes : $\mathrm{K}=1,2$ (valeur moyenne) et $\mathrm{K}=2,4$ (valeur maximale). Le Tableau 1 présente les résultats obtenus sur site, avec une quantité d'explosif égale à $22,5 \mathrm{~kg}$. On a pris des valeurs de la constante $\mathrm{K}$ supérieures à celles indiquées ci-dessus afin d'évaluer son influence sur la vitesse de vibration.

Tableau 1. Vitesses de vibration en $(\mathrm{m} / \mathrm{s})$ à partir de la distance du front de taille [4]

\begin{tabular}{llllllll}
\hline \multicolumn{7}{c}{ Distance $(\mathbf{m})$} \\
\hline & $\mathbf{4 0}$ & $\mathbf{5 0}$ & $\mathbf{6 0}$ & $\mathbf{7 0}$ & $\mathbf{8 0}$ & $\mathbf{9 0}$ & $\mathbf{1 0 0}$ \\
\hline $\mathrm{K}=1,2$ & 0,12 & 0,08 & 0,06 & 0,04 & 0,037 & 0,030 & 0,024 \\
$\mathrm{~K}=1,4$ & 0,15 & 0,10 & 0,07 & 0,05 & 0,043 & 0,035 & 0,029 \\
$\mathrm{~K}=1,6$ & 0,17 & 0,11 & 0,08 & 0,06 & 0,049 & 0,040 & 0,033 \\
$\mathrm{~K}=1,8$ & 0,19 & 0,13 & 0,09 & 0,07 & 0,05 & 0,048 & 0,037 \\
$\mathrm{~K}=2$ & 0,21 & 0,14 & 0,10 & 0,078 & 0,062 & 0,05 & 0,04 \\
$\mathrm{~K}=2,2$ & 0,23 & 0,15 & 0,11 & 0,08 & 0,068 & 0,055 & 0,045 \\
$\mathrm{~K}=2,4$ & 0,25 & 0,17 & 0,12 & 0,09 & 0,07 & 0,06 & 0,049 \\
$\mathrm{~K}=2,6$ & 0,28 & 0,18 & 0,13 & 0,10 & 0,08 & 0,065 & 0,05 \\
$\mathrm{~K}=2,8$ & 0,3 & 0,2 & 0,14 & 0,12 & 0,09 & 0,075 & 0,06 \\
$\mathrm{~K}=3$ & 0,32 & 0,21 & 0,15 & 0,11 & 0,086 & 0,07 & 0,058 \\
\hline
\end{tabular}

En examinant les résultats obtenus sur la Figure 3, on déduit que pour les valeurs les plus probables de la constante $\mathrm{K}(1,6$ à 1,8$)$ les vitesses de vibration atteignent 0,17 à $0,19 \mathrm{~m} / \mathrm{s}$ quand la distance du front de taille est égale à $40 \mathrm{~m}$ et elles descendent à des valeurs proches de $0,04 \mathrm{~m} / \mathrm{s}$ pour la distance maximale de $100 \mathrm{~m}$.

A partir d'un tir, une fraction de l'énergie cédée au terrain se propage sous forme vibratoire, tant dans les fluides (air, eau) que dans les solides. Cette vibration transitoire sollicite les structures sur son passage et $\mathrm{y}$ déclenche divers types de vibrations. Lorsque les efforts engendrés par ces vibrations excédent localement la résistance de ces structures, il peut y avoir apparition de dommages c'est-à-dire la rupture de la roche [3]. On retiendra à ce sujet les propositions qui nous semblent intéressantes :

- toutes les limites ont un caractère probabiliste ;

- les seuils inferieurs à $1 \mathrm{~cm} / \mathrm{s}$, tel qu'ils ont pu être proposés dans certains projets de norme, ne semblent pas être à retenir, la probabilité d'apparition de dégâts étant alors très réduite ;

- la zone de 1 à $3 \mathrm{~cm} / \mathrm{s}$ constitue une fourchette applicable aux immeubles courants habités et aux chantiers de travaux publics classiques.

- à partir de $3 \mathrm{~cm} / \mathrm{s}$, on ressent intensément les vibrations.

En examinant le tracé du tunnel de Dar-El-Oued on a constaté l'absence des édifices privés ou publique, qui peuvent être menacés par les vibrations engendrées par l'usage d'explosif, au voisinage de l'ouvrage réalisé. Une seule exception été faite pour les grottes (un site touristique important) qui se trouve à une distance effective de $200 \mathrm{~m}$ de la cavité du tunnel. Pour cette raison particulière, la vitesse de vibration a été diminuée.

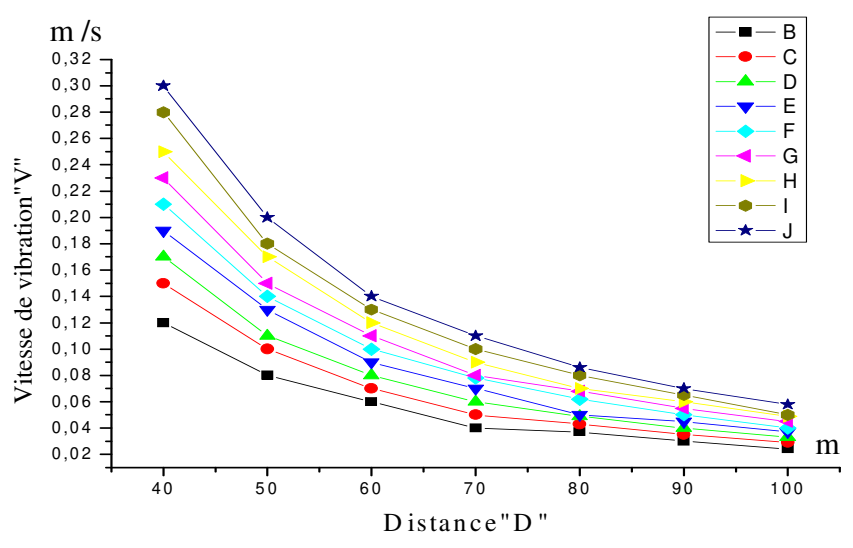

Fig. 3. Influence de la vitesse de vibration en fonction de la distance du front de taille [4]

\section{Mesures de convergence}

Les mesures les plus fréquentes effectuées dans le cas des tunnels sont les mesures de convergence entre plots scellées aux parois. On mesure, généralement à l'aide d'un fil invar maintenu à une tension constante par un dynamomètre, le rapprochement de deux points situés sur des parois opposées de l'ouvrage.

Comme le déplacement d'un point de la paroi intègre la déformation de l'ensemble du massif, les résultats ne sont ainsi pas trop dispersés. C'est pourquoi les mesures de convergence constituent le moyen de contrôle par excellence $\mathrm{du}$ bon comportement d'une galerie souterraine. Le suivi des résultats de l'auscultation en fonction de l'éloignement du front de taille permet de juger la bonne adéquation du soutènement mis en place aux exigences de déformation prévues dans le projet.

Dans le tunnel de Dar El-Oued, des mesures de convergence ont été réalisées, elles consistent à relever et à rendre sous forme graphique et numérique les déplacements dans le plan transversal du tunnel (5 points de mesures pour chaque station) comme le montre la Figure 4.

L'évolution de la déformation relative d'une zone de terrain dans le temps est l'indication fondamentale des courbes de convergence. Des valeurs stables dans le temps indiquent une stabilité mécanique de l'ouvrage, même si ces valeurs sont importantes.

Une convergence négative apparaît durant les premiers jours d'ouverture du terrassement de la section, mais elle reste faible en moyenne pendant les sept premiers jours. Sur certains profils, la stabilisation s'amorce naturellement, il faut noter que cette stabilisation ne peut être obtenue qu'après fermeture de la section (bétonnage des banquettes et du radier).

Les mesures d'auscultation réalisées consistent à relever et à restituer sous forme graphique et numérique l'état des contraintes entre les revêtements provisoire et définitif, sous le pied des cintres, à travers des capteurs de charge et des extensomètres. 
En particulier, les efforts sur le cintre sont mesurés avec des tiges du type à corde vibrante placées sur les ailes, dans le béton de revêtement définitif avec des tiges placées sur l'extrados et sur l'intrados de la section surveillée.

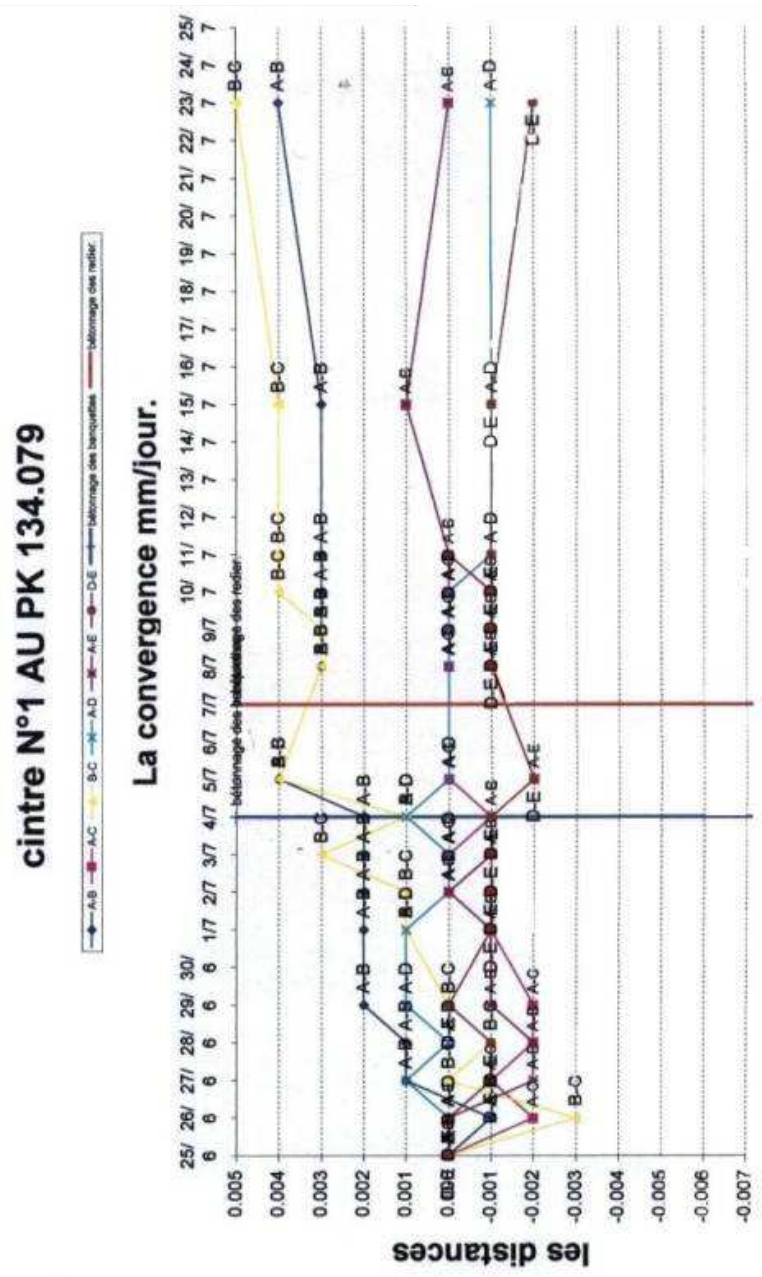

Fig. 4. Mesures de convergence sur le cintre $\mathrm{n}^{\circ} 1$ au PK 134,079 [4]

L'amplitude des déplacements prévisibles est déterminée par les calculs effectués au stade du projet. On retient rarement une valeur maximale admissible pour les déplacements, car l'amplitude des déplacements mesurés ne permet pas à elle seule de juger la stabilité de l'excavation. La vitesse de déplacement est maximale au passage du front dans le plan de mesure. On peut admettre que le déplacement journalier admissible est de l'ordre de $1 / 4$ du déplacement total prévu au passage du front et doit descendre du 1/20 après un délai de l'ordre d'une semaine.

Dans ce type de projet, l'accélération est le critère de base utilisé pour l'interprétation des mesures de déplacement. Généralement, il est admis qu'une accélération des déformations conduit à la rupture. D'autre part, l'influence du soutènement doit également être prise en compte pour une bonne interprétation de son installation et son renforcement. Une décélération non marquée ne peut survenir qu'après un ou deux jours du passage du front.
Les calculs sur le modèle numérique on été effectués au moyen du logiciel SAP 2000, en schématisant la section du tunnel par des tiges rectilignes soumises aux valeurs des pressions déduites à partir des expressions de Terzaghi $[1,2]$ :

- Pression verticale :

$\mathbf{P}_{\mathbf{v}}=\frac{B\left(\gamma-\frac{2 C}{B}\right)}{2 \tan \theta} \cdot\left[1-e^{\frac{-2 \cdot H \tan \theta}{B}}\right]$

- La poussée latérale :

$\mathbf{P}_{\mathbf{h}}=0,3 \cdot \gamma\left(0,5 m+h_{p}\right)$

Le résultat des pressions sur les trois sections étudiées sont résumés dans le Tableau 2 :

Tableau 2. Valeurs des pressions calculées

\begin{tabular}{|c|c|c|}
\hline Section & $\begin{array}{c}\text { Pression } \\
\text { verticale }\left(\mathbf{t} / \mathbf{m}^{\mathbf{2}}\right)\end{array}$ & $\begin{array}{c}\text { Pression } \\
\text { latérale }\left(\mathbf{t} / \mathbf{m}^{\mathbf{2}}\right)\end{array}$ \\
\hline Section 1 & 41,79 & 8,9 \\
\hline Section 2 & 20,80 & 9,77 \\
\hline Section 3 & 20,51 & 9,34 \\
\hline
\end{tabular}

En ce qui concerne le revêtement définitif, les déformations de la coupe 2 étaient moins intenses que celle des coupes 1 et 3 représentée dans la Figure 5. On remarque que ces déformations se concentrent au niveau des piédroits, du radier et beaucoup plus au niveau de la calotte. Les valeurs de ces déformations diminuent lors de la mise en place du soutènement provisoire. D'autre part, on à constaté que le terrain est moins déformable dans la section 2 constitué par une dolomie massive. La plus part des déplacements sont situées en clé de la voûte et en radier. Au final, et après avoir réalisé le soutènement définitif (section entièrement soutenue), les déplacements et les déformations sont moins importantes au niveau de la section 2 par rapport aux sections 1 et 3 .

\section{Conclusion}

A partir de cette étude, on peut conclure que dans la construction des tunnels, les déplacements en surface du sol sont plus faibles par rapport à ceux déterminés au niveau de la voûte et du radier.

Aussi, on note la bonne concordance entre les résultats prévus par notre modèle numérique et les résultats des mesures obtenues sur le site du tunnel. Pour cela et pour assurer la stabilité de l'ouvrage, l'exécution du radier qui s'oppose à la pression ascendante est indispensable.

Sur site, la convergence est mesurée durant toute la période de réalisation du tunnel, quel que soit la position du front de taille (même en position d'arrêt des travaux), 
d'autant plus que la convergence est fonction de deux temps : le temps de creusement (vitesse de creusement et délais de mise de soutènement) et le temps caractéristique du comportement différé du terrain (relaxation).

Enfin, une connaissance de la convergence finale est très intéressante pour prévoir le comportement différé du terrain.

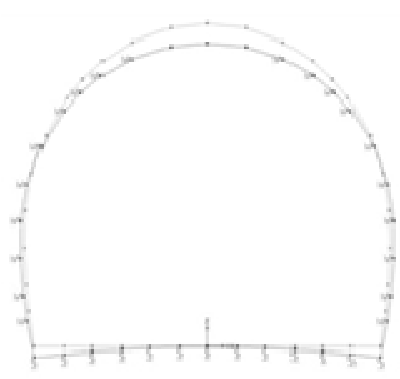

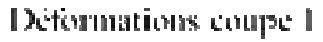

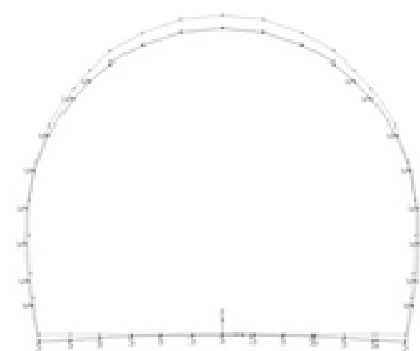

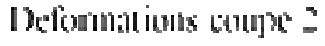

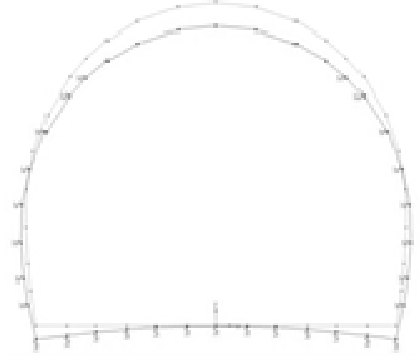

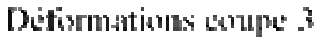

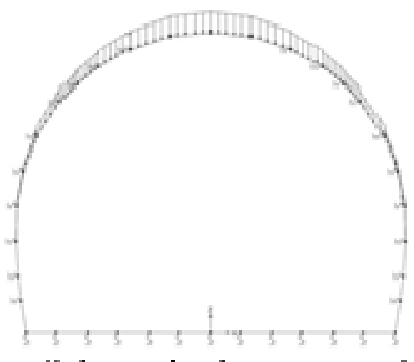

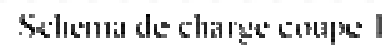

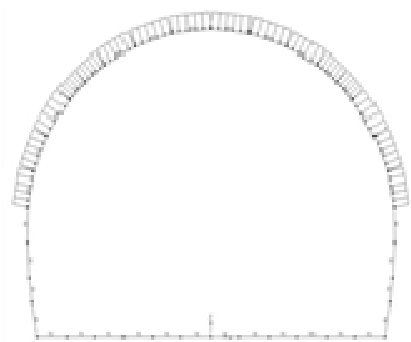

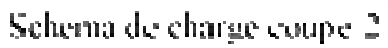

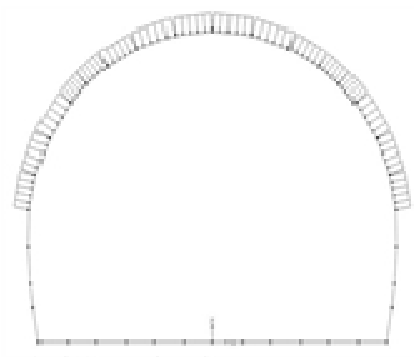

Schena sle charse compe

Fig. 5. Représentation des déformations sur les trois sections étudiées

\section{Références}

[1] A. Bouvard, G. Colombet, Esteulle, F. Ouvrage souterrain : Conception, Réalisation, Entretien, Presse

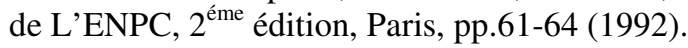

[2] K. Széchy. The art of tunneling. Akadémiai Kiadó, 1097p (1973).

[3] P. Chapot, Etude des vibrations provoquées par les explosifs dans les massifs rocheux, Rapport de recherche LPC $n^{\circ} \mathbf{1 0 5}$ (1981).

[4] C. Yellas, Etude géologique et géotechnique du Tunnel de Oued Dar-El-Oued (Grottes Merveilleuses) Wilaya de Jijel, Mémoire de Magister, Université de Jijel- Algérie (2008). 